\title{
Comparison of the Nucleotide Sequence of Cloned Osteopontin from Hanwoo and Holstein
}

\author{
Tae Young Lee ${ }^{1,2}$, Sung $\mathrm{Kyu} \mathrm{Ju}^{3}$, and Myoung Soo Nam${ }^{1 *}$ \\ ${ }^{1}$ Department of Animal Biosystem Science, Chungnam National University, Daejeon 305-764, Korea \\ ${ }^{2}$ Viral Infectious Disease Research Center, Korea Research Institute of Bioscience and Biotechnology, \\ Daejeon 305-333, Korea \\ ${ }^{3}$ Division of Life Sciences, Chungnam National University, Daejeon 305-764, Korea
}

\begin{abstract}
Osteopontin (OPN) is a secreted phosphorylated glycoprotein. It has an important role in multiple biological processes including cell survival, bone remodeling, inhibition of ectopic calcification, as well as, is thought to have potential immune modulation activities. In this work, we isolated and characterized a full-length open reading frame (ORF) of Korean native cow's OPN from Korean native cow's (Hanwoo) kidney, and successfully cloned firstly on Hanwoo's OPN. The sequencing results indicated that the isolated cDNA was $1190 \mathrm{bp}$ in length containing a complete ORF of $837 \mathrm{bp}$. It encoded a precursor protein Hanwoo's OPN consisting of 278 amino acids with a signal peptide of 16 amino acids. Amino acid homology was found to be $99.3 \%$ as compared to the corresponding sequences of Holstein bone marrow OPN. Hanwoo's kidney OPN and Holstein bone marrow OPN are different only in two amino acid residues 42 and 56, amino acid residue 42 is $\mathrm{Thr}$ (T) $\leftrightarrow \mathrm{Ile}$ (I), and amino acid residue 56 is Ala (A) $\leftrightarrow$ Thr (T) respectively. These results from the present work would be helpful to elucidate the biological function of Hanwoo's OPN and provided a foundation for further insight into role of Hanwoo's OPN.
\end{abstract}

Key words: Korean native cow (Hanwoo), osteopontin, cloning, amino acid

\section{Introduction}

Osteopontin (OPN) is an acidic, phosphorylated glycoprotein of $\mathrm{M}_{\mathrm{r}} 60,000$, secreted in body fluids (e.g., plasma, urine, and milk) and in mineralized tissues. Its expression is increased in many transformed cells and in normal cells exposed to various cytokines (Denhardt and Guo, 1993; Oldberg et al., 1986). OPN is produced by activated $\mathrm{T}$ cells and is implicated in several aspects of immune cell functions, including stimulation of IgG production by B cells (Patarca et al., 1993; Weber and Cantor, 1996). OPN contains a conserved Arg-Gly-Asp sequence, and binds to cells via integrin-mediated mechanisms such as the $\mathrm{a}_{\mathrm{v}} \mathrm{b}_{3}$ as well as the $a_{v} b_{5}$ and $a_{v} b_{1}$ integrins (Liaw et al., 1995). OPN was first isolated from bone and is also present in physiological fluids such as serum, urine (Kohri et al., 1992; Kohri et al., 1993; Shirage et al., 1992) and breast milk (Senger et al., 1989; Sorensen et al., 1993). OPN acts

*Corresponding author: Myoung Soo Nam, Department of Animal Biosystem Science, Chungnam National University, Daejeon 305-764, Korea. Tel: 82-42-821-5782, Fax: 82-42-8232766, E-mail: namsoo@cnu.ac.kr as an opsonin that enhances bacterial phagocytosis (Schack et al., 2009) and plays a pivotal role in the development and maintenance of immune responses (Wang and Denhardt, 2008). OPN induces the NFKB-mediated proMMP-2 activation through IKK-regulated phosphorylation of IKB $\alpha$ and curcumin inhibits OPN-induced cell migration, tumor growth, and NFKB-mediated MMP-2 activation by inhibiting signal leading to IKK activity (Philip and Kundu, 2003).

In human milk, OPN were highly expressed at both mRNA and protein levels during lactation (Nagatomo et al., 2004), and studies proposed that it might be useful in preventing rotavirus infections during lactating period (Naficy et al., 1999). OPN is also present in bovine milk with a concentration of approximately $18 \mathrm{mg} / \mathrm{L}$, which is considerably lower than the corresponding OPN concentrations in human breast milk (approximately $138 \mathrm{mg} / \mathrm{L}$ ) (Bayless et al., 1997; Nagatomo et al., 2004). When the bovine OPN was used as a substrate for transglutaminase, it revealed the presence of two reactive acceptor glutamins (Gln-34 and Gln-316) (Sorensen et al., 1994). Also Sorensen et al. (2003) carried out purification and characterization of osteopontin from human milk. 
In this work, we isolated and characterized a full length open reading frame (ORF) of OPN cDNA from kidney of Korean native cow's (Hanwoo). These results would be helpful to elucidate the biological function of Korean native cow's (Hanwoo) OPN and will also provide a basis for genetic engineering studies of Korean cow's (Hanwoo) OPN.

\section{Materials and Methods}

\section{Molecular cloning and sequencing of the OPN cDNA from Hanwoo's kidney}

Hanwoo's kidney was gained from slaughterhouse and total RNA was separated by guanidium/acidic phenol extract method (Chomczynski and Sacchi, 1987). Protein and DNA fractions were extracted from the homogenate by acidic phenol ( $\mathrm{pH} 4.0$ ) extraction. The upper aqueous phase was transferred into a new reaction tube to precipitate RNA by the addition of 2.5 vol. absolute ethanol. The purity of the RNA was determined in a denaturing MOPSbuffered $1.5 \%$ agarose gel. RNA concentration was measured using spectrophotometer. cDNA was synthesized using oligo-dT primers (Promega, USA) and Superscript II + reverse transcriptase (Life Technologies Inc., USA) from purified RNA. The cDNA encoding the whole open reading frame $(\mathrm{ORF})$ contained signal sequence for the Korean native cow's OPN.

cDNA was amplified by PCR using the following primer. A sense primer (OPN-up) is 5'-GGATCCATGAGAATTGCAGTGATTTG-3', and an antisense primer (OPN-down) is 5'-AAGCTTTCATAGTGACATCAAATTTT -3' resulted in an approximately $1190 \mathrm{bp}$ fragment. Sequence and primer information of the bovine OPN genome were retrieved from GenBank (no. M66236). PCR was performed with $P f u$ DNA polymerase (Stratagene) for 35 cycles as follows: denaturation at $94^{\circ} \mathrm{C}$ for $1 \mathrm{~min}$, annealing at $55^{\circ} \mathrm{C}$ for $1 \mathrm{~min}$, and elongation at $72^{\circ} \mathrm{C}$ for $1 \mathrm{~min}$ $30 \mathrm{~s}$. The PCR products were separated by $1.5 \%$ agarose gel electrophoresis, excised from the gel, purified using the Gel Extraction kit (Qiagen, Switzerland). The amplified fragment was inserted into the Blunt II site of pCRBlunt II-TOPO cloning vector (Invitrogen, USA) according to the manufacturer's instructions. All primers were purchased from Genotech Ltd. (Korea). Unambiguous sequences of 1190 nucleotides of the OPN cDNA were determined using the ABI PRISM cycle sequencing method (Perkin Elmer, USA).

\section{Sequence analysis of OPN}

We performed the alignment and amino acid translation of nucleotide sequences of OPN using the web site software (http://web.expasy.org).

\section{Nucleotide sequence Accession Numbers}

The sequence of the Korean cow's (Hanwoo) OPN was submitted to GenBank under accession number AF492837.

\section{Results and Discussion}

\section{Cloning of OPN}

As shown in Fig. 1, the OPN cDNA were obtained by PCR using a Korean native cow's (Hanwoo) kidney DNA as a template. Expected PCR product in lane 1 having size of $1190 \mathrm{bp}$ corresponded to mature form of OPN (Fig. 1A). These PCR products were inserted into the Blunt II site of pCR-Blunt II-TOPO vector and transformed into the E. coli strain (DH5a) on LB/Kana agar plate. The procedure of cloning the OPN cDNA into this vector schematically showed in Fig. 1B.

\section{Properties of Hanwoo's OPN}

As shown in Fig. 2, Korean native cow's kidney and Holstein bone marrow OPN (GenBank: M66236) amino acid sequence were compared to DNA sequences from NCBI database. Signal peptides (1-16) of Korean native cow's kidney OPN and Holstein bone marrow OPN marked on bold letters [Met (M)-Arg (R)-Ile (I)-Ala (A)Val (V)-Ile (I)-Cys (C)-Phe (F)-Cys (C)-Leu (L)-Leu (L)Gly (G)-Ile (I)-Ala (A)-Ser (S)-Ala (A)]. Korean native cow's (Hanwoo) kidney OPN and Holstein bone marrow

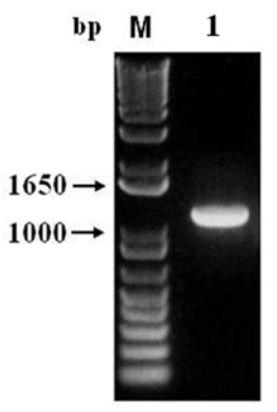

A

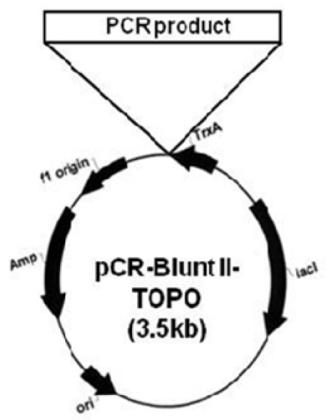

B
Fig. 1. Agarose gel electrophoresis of Korean-native cow's (Hanwoo) OPN. (A) cDNA encoding the mature form of OPN was amplified by PCR. PCR product were electrophoresed on $\mathbf{1 . 2 \%}$ agarose gel. M, DNA marker; lane 1, PCR product of Korean native cow's (Hanwoo) kidney OPN (B) Schematic representation of the OPN cDNA cloning vector. cDNA encoding the mature form of OPN was amplified by RT-PCR and cloned into the pCR-Blunt II-TOPO vector. 


\title{
DNA Sequence
}

\begin{abstract}
ATGAGAATTGCAGTGATTTGCTTCTGCCTCTTGGGCATTGCCTCCGCCCTTCCAGTTAAACCGA
CCAGTTCTGGCAGCTCTGAGGAAAAGCAGCTTAACAACAAATACCCAGATGCTGTAGCCACATGG CTAAAGCCTGACCCATCTCAGAAGCAGACTTTCCTAGCACCACAGAATTCTGTGTCCTCTGAGGAA ACTGATGACAACAAACAAAATACCCTCCCAAGTAAGTCCAATGAAAGCCCTGAGCAAACAGACGA TCTAGATGACGATGATGATAACAGCCAGGACGTCAACTCTAATGACTCCGACGACGCTGAAACCAC TGATGACCCTGACCATTCCGACGAGTCTCACCATTCTGATGAATCTGATGAAGTTGATTTTCCCACT GATATTCCAACAATCGCAGTTTTCACTCCGTTTATCCCTACGGAAAGCGCAAATGATGGCCGAGGTG ATAGTGTGGCTTACGGACTGAAGTCAAGATCTAAGAAGTTCCGCCGATCTAACGTTCAGAGTCCAG ATGCCACAGAGGAGGACTTCACATCACACATAGAGAGTGAGGAGATGCATGACGCACCTAAGAAG ACGAGTCAGCTGACTGACCACAGCAAGGAAACCAACAGTAGCGAGCTTTCCAAAGAACTCACGCC AAAGGCCAAGGATAAGAACAAGCATTCCAATCTGATTGAGAGTCAGGAAAATTCCAAACTCAGCC AAGAATTCCATAGCCTTGAAGACAAGCTAGACCTAGATCATAAGAGTGAAGAAGACAAACACCTG AAAATTCGTATTTCTCATGAATTAGATAGTGCCTCTTCTGAGGTCAATTGA
\end{abstract}

\section{$\underline{\text { Amono Acid Sequence Alignment }}$}

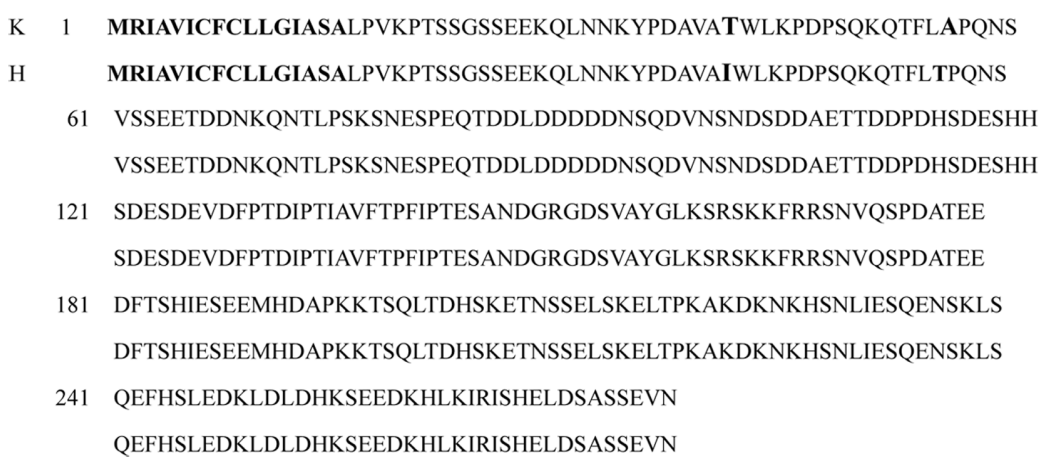

Fig. 2. Comparison of Korean native cow's (Hanwoo) kidney and Holstein bone marrow OPN amino acids. The amino acid sequence corresponding to the mature processed form of Korea native cow's (Hanwoo) kidney OPN. Signal peptides marked on bold letters (1 16). Amino acid sequences differences from the Holstein DNA are bold letter. K : Korean native cow's (Hanwoo) kidney OPN, H : Holstein bone marrow OPN

OPN were different only in two amino acid residues 42 and 56, amino acid residue 42 is $\operatorname{Thr}(\mathrm{T}) \leftrightarrow$ Ile $(\mathrm{I})$, and amino acid residue 56 is Ala (A) $\leftrightarrow \mathrm{Thr}(\mathrm{T})$ respectively (bold letter). Overall, the sequence identity was $99.3 \%$ in comparison to the Holstein bone marrow OPN.

These findings suggest that cloning of Korean native cow's (Hanwoo) kidney OPN can make the expression from eukaryotic cell such as insect cell or mammalian cell. In addition, these results would be helpful to elucidate the biological function of Korean native cow's (Hanwoo) OPN and may be utilized in foodstuffs due to its active biological function.

\section{Acknowledgements}

This study has been supported by the project (20101677) from Chungnam National University.

\section{References}

1. Bayless, K. J., Davis, G. E., and Meininger, G. A. (1997) Isolation and biological properties of osteopontin from bovine milk. Protein Expr. Purific. 9, 309-314.

2. Chomczynski, P. and Sacchi, N. (1987) Single-step method of RNA isolation by acid guanidinium thiocyanate-phenolchloroform extraction. Anal. Biochem. 162, 156-159.

3. Denhardt, D. T. and Guo, X. (1993) Osteopontin: A protein with diverse functions. FASEB J. 7, 1475-1482.

4. Kohri, K., Nomura, S., Kitamura, Y., Nagata, T., Yoshioka, K., Iguchi, M., Yamate, T., Umekawa, T., Suzuki, Y., and Shinohara, H. (1993) Structure and expression of the mRNA encoding urinary stone protein (osteopontin). J. Biol. Chem. 268, 15180-15184.

5. Kohri, K., Suzuki, Y.,Yamamoto, K., Amasaki, N., Yamata, T., Umekawa, T., Lguchi, M., Sinohara, H., and Kurita, T. (1992) Molecular cloning and sequencing of cDNA encoding urinary stone protein, which is identical to osteopontin. Biochem. Biophys. Res. Commun. 184, 859-864. 
6. Liaw, L., Skinner, M. P., Raines, E. W., Ross, R., Cheresh, D. A., Schwartz, S. M., and Giachelli, C. M. (1995) The adhesive and migratory effects of osteopontin are mediated via distinct cell surface integrins. Role of $\mathrm{a}_{\mathrm{v}} \mathrm{b}_{3}$ in smooth muscle cell migration to osteopontin in vitro. J. Clin. Invest. 95, 713724.

7. Naficy, A. B., Abu-Elyazeed, R., and Holmes, J. L. (1999) Epidemiology of rotavirus diarrhea in Egyptian children and implications for disease control. Am. J. Epidemiol. 150, 77777.

8. Nagatomo, T., Ohga, S., Takada, H., Nomura, A., Hikino, S., Imura, M., Ohshima, K., and Hara, T. (2004) Microarray analysis of human milk cell: Persistent high expression of osteopontin during the lactation period. Clin. Exp. Immuno. 138, 47-52.

9. Oldberg, A., Franzen, A., and Heinegard, D.(1986) Cloning and sequence analysis of rat bone sialoprotein(osteopontin) cDNA reveals an Arg-Gly-Asp cell-binding sequence. Proc. Natl. Acad. Sci. U.S.A. 83, 8819-8823.

10. Patarca, R., Saavedra. R., and Cantor, H. (1993) Molecular and cellular basis of genetic resistance to bacterial infection: the role of the early T-lymphocyte activation-1/osteopontin gene. Crit. Rev. Immunol. 13, 225-246.

11. Philip, S. and Kundu, G. C. (2003) Osteopontin induces nuclear factor kappa B-mediated promatrix metalloproteinase2 activation through I kappa B alpha/IKK signaling pathways, and curcumin (diferulolylmethane) down-regulates these pathways. J. Biol. Chem. 278, 14487-14497.

12. Schack, L., Stapulionis, R., Christensen, B., Kofod-olsen, E., Skov Sorensen, U. B., Vorup-Jensen, T., Sorensen, E. S., and
Hollsberg, P. (2009) Osteopontin enhances phagocytosis through a novel osteopontin receptor, the alphaXbeta2 integrin. J. Immunol. 182, 6943-6950.

13. Senger, D. R., Peruzzi, C. A., Papadopoulos, A., and Tenen, D. G. (1989) Purification of a human milk protein closely similar to tumor-secreted phosphoproteins and osteopontin. Biochem. Biophys. Acta 996, 43-48.

14. Shiraga, H., Min, W., VanDusen, W. J., Clayman, M. D., Miner, D., Terrell, C. H., Sherbotie, J. R., Foreman, J. W., Przysiecki, C., Neilson, E. G., and Hoyer, J. R. (1992) Inhibition of calcium oxalate crystal growth in vitro by uropontin, a new member of the aspartic acid-rich protein superfamily. Proc. Natl. Acad. Sci. U.S.A. 89, 426-430.

15. Sørensen, S., Justesen, S. J., and Johnsen A. H. (2003) Purification and characterization of osteopontin from human milk. Protein Expr. Purific. 30, 238-245.

16. Sørensen E. S. and Petersen, T. E. (1993) Purification and characterization of three proteins isolated from the proteose peptone fraction of bovine milk. J. Dairy Res. 60, 189-197.

17. Sørensen, E. S., Rasmussen, L. K., Moller, L., Jensen, P. H., Hojrup, P., and Petersen, T. E. (1994) Localization of transglutaminase-reactive glutamine residues in bovine osteopontin. Biochem. J. 304, 13-16.

18. Wang, K. X. and Denhardt, D. T. (2008) Osteopontin: Role in immune regulation and stress responses. Cytokine Growth Factor Res. 19, 333-345.

19. Weber, G. F. and Cantor, H. (1996) The immunology of Eta1/osteopontin. Cytokine Growth Factor Res. 7, 241-248.

(Received 2013.2.3/Revised 2013.5.10/Accepted 2013.5.16) 